\title{
Abordagem fuzzy para caracterização de incertezas estruturais e quantificação de riscos hidrológicos: estudo de caso do reservatório Orós no semiárido brasileiro
}

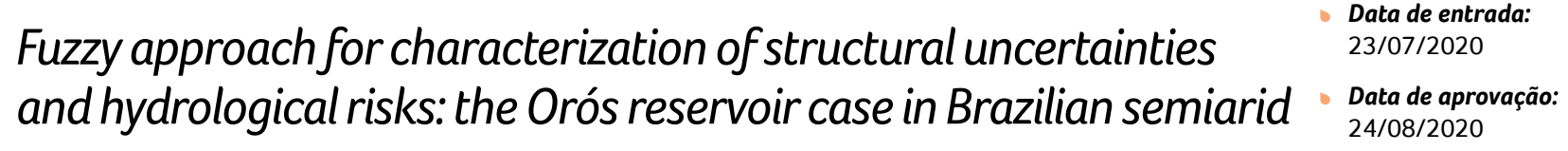

João Batista de Sousa Raulino ${ }^{1 * *}$ | Vanda Tereza Costa Malveira² | Iran E. Lima Neto

DOI: https://doi.org/10.36659/dae.2022.013

ORCID ID

RaulinoJBS (D) https://orcid.org/0000-0002-6119-9565
Malveira VTC (D) https://orcid.org/0000-0002-7002-1240

Lima Neto IE (iD https://orcid.org/0000-0001-8612-5848

\section{Resumo}

Nos estudos de Recursos Hídricos, a identificação e a caracterização das incertezas são etapas necessárias e importantes da modelagem dos sistemas hídricos, pois permitem ter um entendimento das interações entre os dados, parâmetros e estrutura do modelo. 0 risco de um sistema depende das incertezas presentes e o quanto elas podem torná-lo vulnerável a solicitações externas. As incertezas são, portanto, o plano de fundo onde os riscos são gerados. Este trabalho teve como objetivo demonstrar a aplicabilidade do Números Fuzzy Triangulares (NFTs) na identificação e caracterização de incertezas hidrológicas em um exemplo simples, bem como utilizá-los para quantificar os riscos do sistema hídrico associado. A metodologia consistiu em identificar e classificar a incerteza na vazão afluente em razão de duas fontes distintas que contrastam quanto à área de drenagem da sub-bacia do reservatório Orós, localizado no Estado do Ceará, Brasil, caracterizá-la utilizando NFTs e quantificar o risco fuzzy associado em usar uma ou outra no processo de geração de informações hidrológicas. Os resultados demonstraram que a incerteza identificada é estrutural, sendo que foi possível caracterizá-la por meio dos elementos característicos do NFT considerando as características de regiões semiáridas. Os riscos calculados demonstram que considerar a vazão afluente fornecida pela Agência Nacional de Águas (ANA) em vez da da Companhia de Gestão dos Recursos Hídricos do Ceará (COGERH) pode afetar a real capacidade do sistema. No exemplo numérico para ilustrar o impacto da incerteza estrutural, vazão regularizada, observou-se um impacto significativo sobre a real capacidade do sistema hídrico. O risco de falha da vazão regularizada, determinada a partir dos dados da ANA, foi $24 \%$ maior dos que os $10 \%$ usados para estimar a vazão regularizada, quando comparado com a vazão regularizada gerada a partir dos dados da COGERH. Os NFTs conseguiram caracterizar e quantificar o risco da incerteza estrutural no sistema estudado, demonstrando que o NFT é uma ferramenta aplicável e útil na obtenção de informações hidrológicas para gerenciamento de recursos hídricos em bacias semiáridas.

Palavras-chave: Números Fuzzy Triangulares (NFT). Incertezas. Risco. Semiárido. Vazão afluente.

\footnotetext{
${ }^{1}$ Universidade Federal do Ceará - Fortaleza - Ceará - Brasil.

2 Universidade Estadual Vale do Acaraú - Sobral - Ceará - Brasil.

*Autor correspondente: jbraulinosagmail.com.
} 


\section{Abstract}

In Water Resources studies, the identification and characterization of uncertainties are important steps in the modeling of water systems, as they allow an understanding of the interactions between data, parameters and model structure. The risk of a system depends on the uncertainties present and how much they can make it vulnerable to external loads. Uncertainties are, therefore, the background where risks are generated. This research aimed to demonstrate the applicability of Triangular Fuzzy Numbers (NFTs) in the identification and characterization of hydrological uncertainties, as well as using them to quantify the risks of the associated water system. The methodology consisted of identifying and classifying the uncertainty in the streamflow due to two different sources that contrast regarding the drainage area of the Orós reservoir sub-basin, located in the State of Ceará, Brazil, characterizing it using NFTs and quantifying the fuzzy risk associated with using one or the other in the process of generating hydrological information. The results showed that the identified uncertainty is a structural uncertainty, and it was possible to characterize it through the characteristic elements of the NFT considering the semi-arid characteristics. The calculated risks demonstrate that considering the streamflow provided by the National Water Agency (ANA) instead of that from the Water Resources Management Company (COGERH) can affect the capacity of the system. In the numerical example to illustrate the impact of structural uncertainty, yield, a significant impact was observed on the real capacity of the water system. The risk of failure of the yield, determined from ANA's data, was $24 \%$ higher than the 10\% used to estimate the yield, when compared to the yield generated from COGERH's data. The FTNs were able to characterize and quantify the risk of structural uncertainty in the studied system, demonstrating that the NFT is an applicable and useful tool in obtaining hydrological information for the Management of Water Resources. Keywords: Triangular Fuzzy Numbers (TFN). Uncertainties. Risk. Semiarid. Streamflow.

\section{INTRODUÇÃO}

Nos estudos de Recursos Hídricos, a identificação e a caracterização das incertezas são etapas relevantes e imprescindíveis da modelagem dos sistemas hídricos, pois permitem ter um entendimento das interações entre os dados, parâmetros e estrutura do modelo (MONTANARI, 2011; SORDO-WARD et al., 2016; AHMADI, NASSERI e SOLOMATINE, 2019; TAGEGNE et al., 2019 ). No entanto, muitas vezes essas etapas são negligenciadas, tendo impacto direto no adequado gerenciamento de sistemas hídricos.

Quando se estuda o tema incerteza em um sistema hídrico, uma das primeiras etapas é diferenciá-la de risco. A literatura segue duas direções contrastantes e, portanto, é imprescindível tomar posição quanto às diferenciações. A primeira abordagem segue a definição que segue a linha de raciocínio de Yevjevich (1983) e considera que as incertezas não possuem natureza quan- tificável. Nesse sentido, o risco pode ser definido como a probabilidade (risco probabilístico) ou a possibilidade (risco fuzzy) de eventos, valores ou fenômenos indesejáveis (VIEIRA, 2005). Na segunda abordagem, a incerteza possui natureza quantificável eo risco mantém a mesma definição da primeira (LIU e GUPTA, 2007; VALLAM, QIN e YU, 2014). Com exceção do conceito de natureza mensurável ou não, a incerteza possui propriedades semelhantes nas duas abordagens: as incertezas podem ser identificadas, caracterizadas e incorporadas nos processos de modelagem dos sistemas hídricos. O objetivo de considerar as incertezas nos modelos é fornecer outputs mais condizentes com a realidade e com a variabilidade intrínseca dos fenômenos naturais.

As incertezas presentes e que permeiam os Recursos Hídricos são classificadas em dois tipos: aleatórias e epistêmicas (GANOULIS, 2009; TUNG, 2018). Os termos naturais e informacio- 
nais também são denominações que costumam aparecer na literatura, sendo sinônimos de incertezas aleatórias e epistêmicas, respectivamente. É importante ter em mente que a incerteza natural não pode ser reduzida, mesmo que um modelo mais "preciso" seja desenvolvido ou que longas séries históricas estejam disponíveis (obtidas com erros não significativos) (CAMPOS, SOUZA FILHO e LIMA, 2014). As incertezas epistêmicas podem ser subdivididas em três grupos: incerteza métrica, incerteza paramétrica e incerteza estrutural. As incertezas métricas são fruto, por exemplo, da imprecisão dos instrumentos de medição no processo de obtenção de dados de precipitação, sendo, portanto, concernentes aos inputs dos modelos. A incerteza paramétrica é relativa às incertezas presentes nos parâmetros dos modelos. Já as incertezas estruturais são oriundas das simplificações e hipóteses dos modelos que buscam representar os fenômenos naturais, portanto relativas às próprias configurações dos modelos. (RENARD et al., 2010; MCMILLAN et al., 2011; ENGELAND et al., 2016; LIU et al., 2017; GAN et al., 2018; GUPTA e GOVINDARAJU, 2019).

De maneira geral, três abordagens principais de identificação e caracterização das incertezas, bem com avaliação de riscos associados, podem ser distinguidas: métodos probabilísticos, métodos possibilísticos e métodos híbridos. Majoritariamente, os métodos probabilísticos caracterizam as incertezas por meio de frequências acumuladas e funções densidade de probabilidade, podendo ser aplicados tanto nos inputs como nos outputs dos modelos. Os métodos possibilísticos utilizam a Teoria dos Conjuntos Fuzzy como uma alternativa ao método probabilístico para lidar com as incertezas presentes nos sistemas naturais, no processo de modelagem e nos modelos. A terceira abordagem é denominada "métodos híbridos". Como a própria denominação sugere, essa abordagem combina métodos probabilísticos e métodos baseados na Teoria dos Conjuntos Fuzzy (AHMADI, NASSERI e SOLOMATINE, 2019). A principal vantagem da abordagem fuzzy e da abordagem Bayesiana, abordagem subjetivista equivalente para os métodos probabilísticos, é a possibilidade de inserção do conhecimento de especialistas no processo da modelagem e nos modelos propriamente ditos. Quando se estudam sistemas complexos, essa propriedade se torna ainda mais relevante e útil. É uma das razões de um grande número de aplicações dessas abordagens nos estudos de incertezas pertencentes aos recursos hídricos (KESKIN, TAYLAN e TERZI, 2006; ZHU e ZHOU, 2008; HUANG et al., 2010; BOUDA et al., 2012; CHACHI, TAHERI e ARGHAMI, 2014; WANG et al., 2015; ZAKHROUF, BOUCHELKIA e STAMBOUL, 2015; WAGENA et al., 2019).

A abordagem fuzzy é uma ferramenta mais adequada do que a probabilística para estudar incertezas epistêmicas, em razão do conhecimento impreciso ou incompleto do sistema real (JACQUIN e SHAMSELDIN, 2007. O Número Fuzzy Triangular (NFT), caso particular dos números fuzzy, é frequentemente usado, pela simplicidade e por representar adequadamente muitos fenômenos hidrológicos. O NFT é definido completamente por três elementos característicos. Esses elementos podem ser definidos com base em dados observados da variável estudada ou por meio da experiência de um especialista sobre fenômeno investigado. Nasseri, Anzari e Zahraie (2014) utilizaram os limites mínimo e máximo e os valores mais frequentes dos parâmetros de um modelo hidrológico para definir a função de pertinência do NFT, a fim de avaliar a propagação das incertezas dos parâmetros aos outputs do modelo hidrológico. Khazaei e Hosseini (2015) utilizaram o conceito de NFT para inserir as incertezas em cada componente da equação do balanço hídrico em nível de bacia hidrográfica, buscando avaliar as incertezas e reduzi-las no cálculo da vazão afluente. Chachi, Taheri e Arghami (2014) utilizaram a regressão linear fuzzy 
para correlacionar a descarga e carga suspensa na água em nível de bacia hidrográfica. Esses autores geraram a informação da carga suspensa de sedimentos por meio de um NFT, com o objetivo de fornecer subsídios para a tomada de decisão em um ambiente com menos incertezas. Embora esses estudos tenham utilizados dados coletados para a concepção da função de pertinência do NFT, este é particularmente útil quando se dispõe de poucos dados ou quando são de difícil mensuração (GANOULIS, 2009).

Quando se usa a abordagem fuzzy, existem dois caminhos a seguir. O primeiro, denominado lógica fuzzy, consiste em três componentes: funções de pertinência do input/output, uma base de regras e um sistema de inferência (MAMDANI, 1974). Essa abordagem é ideal para propor modelos para a descrição de sistemas complexos. No segundo, define-se a capacidade e a solicitação como fuzzy a fim de incorporar as incertezas que permeiam essas propriedades e, consequentemente, determinar o risco que o sistema experimenta. $O$ risco de um sistema depende das incertezas presentes e o quanto elas podem torná-lo vulnerável a solicitações. As incertezas são, portanto, o plano de fundo onde os riscos são gerados (VIEIRA, 2005). Todo sistema está exposto a ações externas de diferentes naturezas. No geral, essas ações são denominadas genericamente de solicitação e tendem a deixar o sistema fora do seu equilíbrio natural. Por outro lado, o sistema responde à solicitação com uma ação de mesma natureza, que é denominada de capacidade do sistema. $\mathrm{O}$ confronto entre capacidade e solicitação indica o desempenho do sistema a um esforço externo (TUNG, YEN e MELCHING, 2005). São exemplos de falhas de um sistema hídrico: um reservatório não consegue fornecer água suficiente para os usos pré-definidos; um vertedouro tem desempenho insatisfatório contra uma cheia; um corpo hídrico possui concentração de poluentes acima dos níveis permitidos pelas legislações ambientais. No primeiro caso, a solicitação é a demanda dos diversos usos e a capacidade é a oferta de água do reservatório. No segundo, a capacidade é a altura da lâmina máxima projetada do vertedouro e a solicitação um evento extremo de vazão afluente, por exemplo, sob cenários de mudanças climáticas. No último, a solicitação é a carga de poluentes oriunda de fontes pontuais e difusas de uma bacia ou sub-bacia hidrográfica e a capacidade seria autodepuração do corpo receptor. A variabilidade natural dos fenômenos e incertezas presentes nos inputs, parâmetros e estrutura dos modelos podem afetar a capacidade e a solicitação, de modo que não podem ser definidas deterministicamente. Portanto, na definição da solicitação e da capacidade, é necessário identificar e caracterizar as incertezas presentes no sistema analisado e, se elas forem relevantes para o fenômeno, devem ser incorporadas na concepção dessas propriedades e, assim, analisar os riscos que elas podem gerar. Uma das maneiras de considerar as incertezas na avaliação do desempenho dos sistemas é por meio de NFTs, como mencionado anteriormente.

\section{OBJETIVO}

Este trabalho teve como objetivos: (1) identificar e classificar a incerteza na vazão afluente em razão de duas fontes distintas (Agência Nacional de Águas - ANA e Companhia de Gestão dos Recursos Hídricos - COGERH) que contrastam quanto à área de drenagem da sub-bacia do reservatório Orós, localizado no estado do Ceará, Brasil; (2) caracterizar a incerteza utilizando Números Fuzzy Triangulares; e (3) quantificar o risco fuzzy associado ao processo de geração de informações hidrológicas. No entendimento dos autores, o presente artigo é o primeiro a abordar esse tipo de problema em bacias semiáridas. 


\section{MATERIAIS E MÉTODOS}

\subsection{Sistema hídrico analisado}

O sistema definido para análise de risco hidrológico utilizando os NFTs foi o reservatório Orós, localizado no Estado do Ceará, Brasil, concentrando-se na fonte de informação da vazão afluente. A incerteza está presente porque a vazão afluente fornecida pela ANA considera a vazão medida no posto fluviométrico de Iguatu como representativa de toda a sub-bacia do reservatório, embora despreze cerca de $20 \%$ da sua área de drenagem (Lima Neto et al., 2011). Já a vazão afluente fornecida pela COGERH é estimada por meio do balanço hídrico do reservatório, portanto considera toda a área de drenagem da sub-bacia no processo. A parcela da área não contabilizada pode contribuir para a geração de escoamento rumo ao Orós, principalmente na estação chuvosa. A Fig. 1 exibe a sub-bacia do Orós, onde é possível verificar a parcela da área não contabilizada pelas medições do posto pluviométrico de Iguatu (seção de controle). O Orós está inserido na sub-bacia cearense do Alto Jaguaribe, pertencente à Bacia do Rio Jaguaribe, a qual ainda se subdivide nas sub-bacias Médio Jaguaribe, Baixo Jaguaribe, Salgado e Banabuiú. A sub-bacia do Médio Jaguaribe é a maior sub-bacia do Estado do Ceará com área de 24639 km² e está totalmente inserida na região semiárida do estado. 0 reservatório Orós é o segundo maior do estado com volume máximo estocável de $1940 \mathrm{hm}^{3}$.

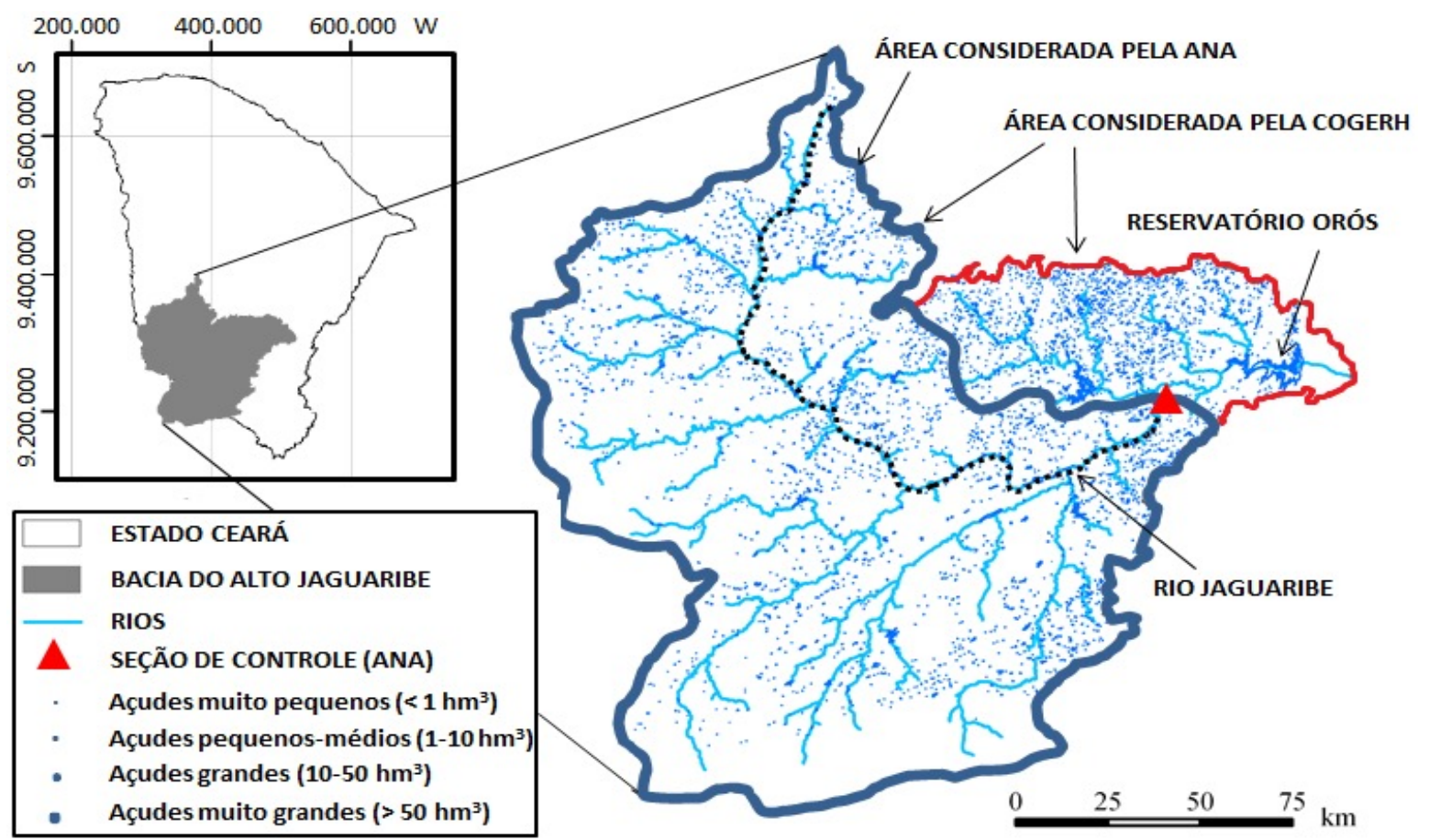

Figura 1 - Localização do posto pluviométrico de Iguatu e do reservatório Orós, localizado no Estado do Ceará, Brasil.

\subsection{Conjuntos fuzzy}

A lógica fuzzy é uma generalização da lógica clássica, traduzidas matematicamente por meio dos conjuntos fuzzy e crisp, respectivamente. $O$ conjun- to fuzzy surgiu da necessidade de representar grandezas que não possuem fronteiras bem definidas, em razão da sua natureza, métodos de mensuração ou conhecimento incompleto (SIMÕES e SHAW, 
2007). Nesse contexto, os conjuntos fuzzy permitem trabalhar com as incertezas, incorporando-as no processo de modelagem de um sistema, diferentemente dos conjuntos crisp, que têm natureza determinística. Um conjunto fuzzy é caracterizado por sua função de pertinência, que associa a cada elemento de um certo conjunto fuzzy um valor no intervalo [0,1]. $O$ valor entre 0 e 1 indica o grau de pertencimento do elemento no conjunto fuzzy definido, ou seja, o quanto o elemento se enquadra no conceito que o conjunto fuzzy está representando. Quanto mais próximo de 1 , mais o elemento pertence ao conjunto e, consequentemente, mais precisas são as informações que se tem sobre o elemento (ZADEH, 1965). Um conjunto fuzzy é matematicamente definido como:

$$
\widetilde{\mathrm{A}}=\left\{\left(\mathrm{x}, \mu_{\widetilde{\mathrm{A}}}(\mathrm{x})\right) / \mathrm{x} \in \mathrm{X}\right\}
$$

em que $\widetilde{A}$ é conjunto fuzzy, $x$ elemento genérico do espaço de pontos $X$ e $\mu_{\widetilde{A}}(\mathrm{X})$ é a função de pertinência, que mapeia o grau de pertencimento de $x$ no conjunto $\widetilde{A}$, denominado de nível ou grau de pertinência. O contradomínio definido por Zadeh (1965) não necessariamente tem que ser o intervalo real $[0,1]$, pode ser qualquer subconjunto de $\mathrm{R}^{+}$(ZIMMERMANN, 2001). Quando o contradomínio é $[0,1]$ e o conjunto fuzzy satisfaz as propriedades de normalidade e convexidade, define-se um caso particular dos Conjuntos Fuzzy, os números fuzzy. A normalidade significa que o valor máximo de pertinência é 1 , enquanto a convexidade pode ser compreendida com a não existência de pontos de mínimo local.

\subsection{Representação da incerteza por meio do NFT}

Como a incerteza da real vazão que alimenta o açude Orós pode ser importante para o processo de geração de informação hidrológica e pode gerar riscos na tomada de decisão, foi representada por um NFT com o objetivo de determinar o risco associado a essa simplificação (estabelecer a vazão da ANA como representativa de toda a sub-bacia). A Fig. 2 exibe a representação gráfica da vazão afluente do açude Orós, e de qualquer reservatório, por um NFT.

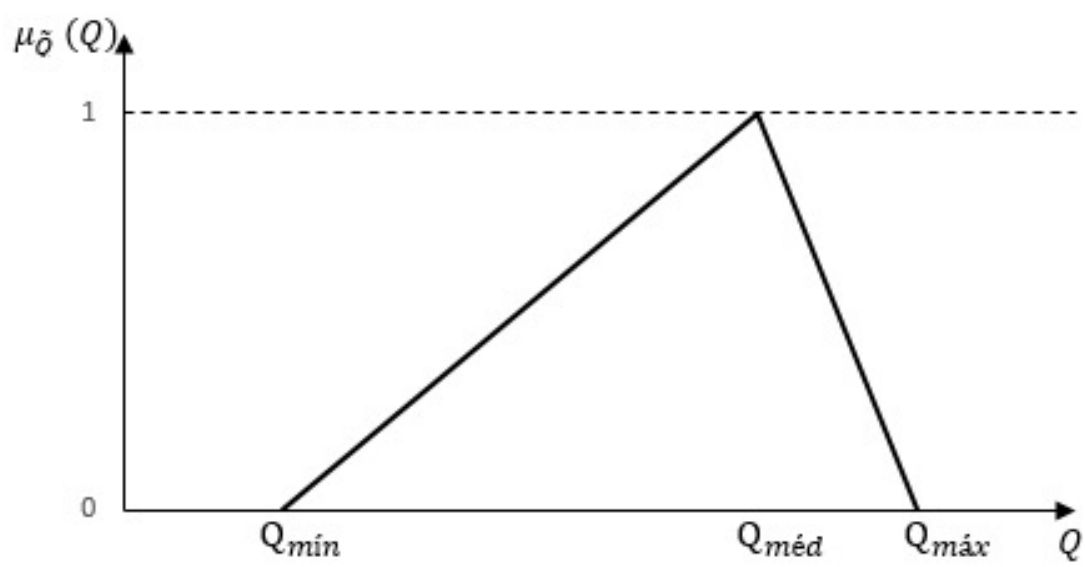

Figura 2 - Representação da incerteza da vazão afluente por um NFT.

Um NFT é representado matematicamente pela seguinte expressão:

$$
\widetilde{\mathrm{Q}}_{\mathrm{a}}=\left(\mathrm{Q}_{\text {mín }}, \mathrm{Q}_{\text {méd }}, \mathrm{Q}_{\text {máx }}\right)
$$

em que $\widetilde{Q}_{a}\left(L^{3} T^{-}\right)$vazão afluente fuzzy, $Q_{\text {min }}\left(L^{3} T^{-}\right)$ extremo esquerdo do domínio do NFT da vazão afluente fuzzy, cujo nível de pertinência é zero, $\mathrm{Q}_{\text {máx }}\left(\mathrm{L}^{3} \mathrm{~T}^{-}\right)$é extremo direito do domínio do NFT da vazão afluente fuzzy, cujo nível de pertinên- 
cia é zero e $\mathrm{Q}_{\text {méd }}\left(\mathrm{L}^{3} \mathrm{~T}\right)$ ), o valor do domínio da vazão afluente fuzzy com máximo nível de pertinência (1).

O elemento $Q_{\text {mín }}\left(\mathrm{L}^{3} \mathrm{~T}^{-}\right)$foi estabelecido de acordo com a percepção de um especialista sobre o padrão de precipitação da bacia do Rio Jaguaribe: na estação chuvosa propriamente dita (fevereiro-maio) foi considerado como a vazão afluente mínima da série histórica do período 1986-2010 e, fora da estação chuvosa, foi considerado como nulo. Essa definição se deu em razão da sazonalidade da vazão afluente do reservatório Orós, resultado da variabilidade temporal e espacial da precipitação, característica da região semiárida a qual a bacia do Alto Jaguaribe está inserida. Por outro lado, os outros dois elementos foram definidos de acordo com os dados disponíveis. A série histórica existente da vazão afluente foi utilizada para determinar o $\mathrm{Q}_{\text {med }}\left(\mathrm{L}^{3} \mathrm{~T}^{-}\right)$, elemento de máxima pertinência, como sendo a vazão média mensal da série histórica, uma vez que o valor da média pode ser encarado como representativo da série histórica da vazão afluente (CAMPOS, SOUZA FILHO e LIMA, 2014). Já o elemento $\mathrm{Q}_{\text {máx }}\left(\mathrm{L}^{3} \mathrm{~T}^{-}\right)$foi considerado como sendo a máxima vazão afluente observada na série histórica. Nesse sentido, as vazões afluentes fornecidas pela ANA e pela COGERH foram definidas como a capacidade e solicitação do sistema, respectivamente, como descrito a seguir. É importante enfatizar que se trata de uma abordagem inédita para representar incertezas na vazão afluente de bacias semiáridas, dentro do contexto da incerteza investigada.

$\widetilde{\mathrm{Q}}_{\mathrm{a}, \mathrm{ANA}}=\left(\mathrm{Q}_{\mathrm{mín}, \mathrm{ANA}}, \mathrm{Q}_{\mathrm{méd}, \mathrm{ANA}}, \mathrm{Q}_{\mathrm{máx}, \mathrm{ANA}}\right)$

$\widetilde{\mathrm{Q}}_{\mathrm{a}, \mathrm{COGERH}}=\left(\mathrm{Q}_{\text {mín,COGERH }}, \mathrm{Q}_{\text {méd,COGERH }}, \mathrm{Q}_{\text {máx,COGERH }}\right)$ em que $\widetilde{Q}_{a, A N A}\left(L^{3} T^{-}\right)$é a vazão afluente fornecida pela ANA representada por um NFT e $\widetilde{\mathrm{Q}}_{\mathrm{a} \text {,COGERH }}\left(\mathrm{L}^{3} \mathrm{~T}^{-}\right)$, a vazão afluente fornecida pela COGERH representada por um NFT.

Os dados de vazão referentes ao posto fluviométrico de Iguatu foram adquiridos no website $<w w w$. snirh.gov.br/hidroweb/> (pertencente à ANA). Os valores da vazão afluente diretamente no reservatório foram adquiridos via solicitação ao Governo do Estado do Ceará, por meio do website <https:// www.cearatransparente.ce.gov.br/>. Com o objetivo de compatibilizar as séries históricas de vazões afluentes entre as duas fontes, foi utilizado o período de 1986-2010 para a realização análise de risco. Já os dados de vazões afluentes do período 20142019 foram usados para verificar os resultados encontrados para os riscos fuzzy mensais.

A função-desempenho fuzzy ( $\widetilde{Z})$ foi definida como a diferença entre a vazão fornecida pela ANA e a vazão fornecida pela COGERH, como mostrado a seguir.

$\widetilde{\mathrm{Z}}=\widetilde{\mathrm{Q}}_{\mathrm{a}, \mathrm{ANA}}-\widetilde{\mathrm{Q}}_{\mathrm{a}, \mathrm{COGERH}}$

Quando a capacidade e a solicitação são representadas por NFTs, a operação básica utilizada para determinar a função-desempenho fuzzy é a subtração de números fuzzy, considerando as propriedades da matemática intervalar. Nesse sentido, foi realizada a definição do oposto da solicitação, Eq. 6, e em seguida a adição dos números fuzzy, Eq. 7 para gerar a função-desempenho fuzzy, Eq. 8. As expressões que determinam essas etapas estão descritas a seguir.

$$
\begin{aligned}
& -\widetilde{\mathrm{Q}}_{\mathrm{a}, \mathrm{COGERH}}=\left(-\mathrm{Q}_{\text {máx,COGERH }},-\mathrm{Q}_{\text {méd,COGERH }},-\mathrm{Q}_{\text {mín,COGERH }}\right) \\
& \tilde{\mathrm{Z}}=\widetilde{\mathrm{Q}}_{\mathrm{a}, \mathrm{ANA}}+\left(-\widetilde{\mathrm{Q}}_{\mathrm{a}, \mathrm{COGERH}}\right)
\end{aligned}
$$


$\tilde{Z}=\left(Q_{\text {mín,ANA }}-Q_{\text {máx,COGERH }}, Q_{\text {méd,ANA }}-Q_{\text {méd,COGERH }}, Q_{\text {máx,ANA }}-Q_{\text {mín,COGERH }}\right)$

No contexto dos números fuzzy, o risco Ké definido como a razão entre a área abaixo da curva da função de pertinência onde o domínio é negativo pela área total abaixo da curva, sendo que a parcela positiva da área representa a confiabilidade (R) do sistema. Matematicamente, o risco e a confiabilidade são definidos, respectivamente, pelas Eq. 9 e 10.

$$
K=\frac{\int_{\mathrm{z} \leq 0} \mu_{\widetilde{\mathrm{Z}}}(\mathrm{z}) \mathrm{dz}}{\int_{\mathrm{z}} \mu_{\widetilde{\mathrm{Z}}}(\mathrm{z}) \mathrm{dz}}
$$

$$
R=1-K=\frac{\int_{z>0} \mu_{\widetilde{Z}}(z) d z}{\int_{z} \mu_{\widetilde{Z}}(z) d z}
$$

em que $z$ é o elemento que representa a variável estudada, no caso as vazões afluentes fornecidas pela ANA e COGERH.

Existem quatro situações distintas de risco fuzzy que uma função-desempenho definida como um NFT pode experimentar, como é mostrado na Fig. 3.

(a)

(b)
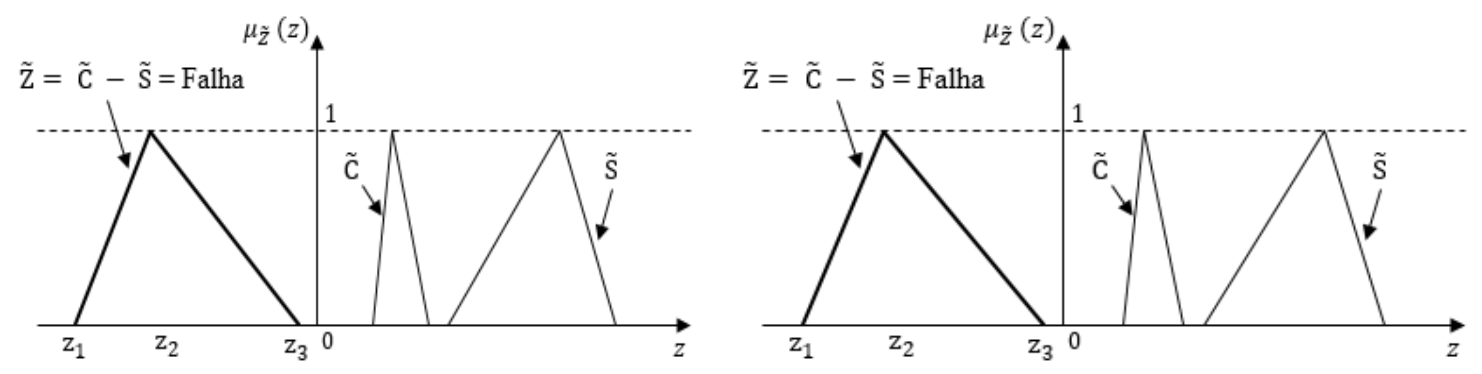

(c)

(d)
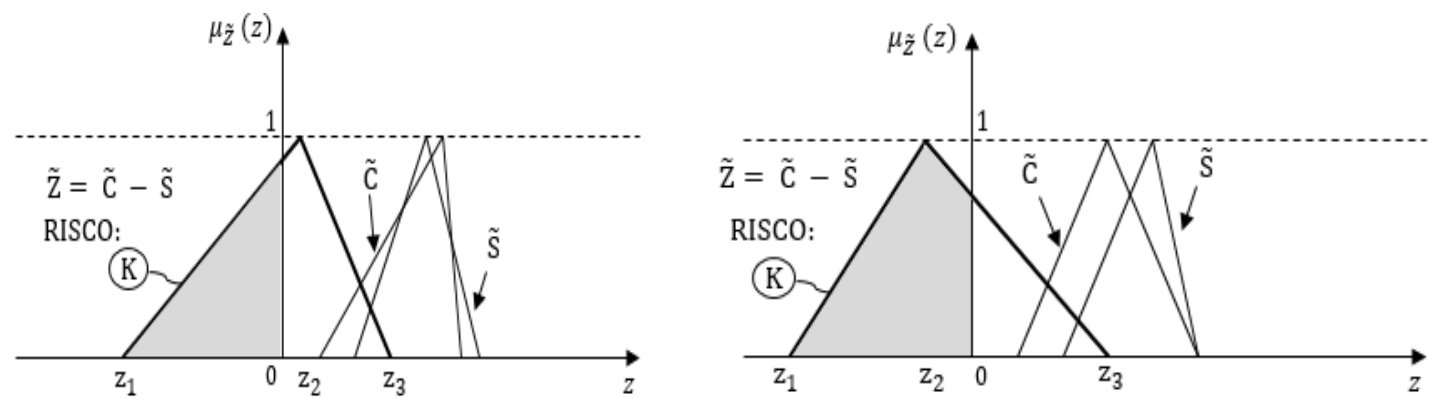

Figura 3 - Possíveis riscos fuzzy de uma função-desempenho representado por um NFT: (a) falha total, (b) segurança total, (c) risco onde a função à esquerda do ponto de máximo do NFT toca o eixo das ordenadas e (d) risco onde a função à direita do ponto de máximo do NFT toca o eixo das ordenadas.

As Eq. 11 e 12 representam a quantificação dos riscos das situações expressas na Fig. 3 (c) e (d), respectivamente.

$$
\mathrm{K}=\frac{\mathrm{z}_{1}^{2}}{\left(\mathrm{z}_{2}-\mathrm{z}_{1}\right)\left(\mathrm{z}_{3}-\mathrm{z}_{1}\right)}
$$




$$
\mathrm{K}=\frac{\left(\mathrm{z}_{3}-\mathrm{z}_{2}\right)\left(\mathrm{z}_{3}-\mathrm{z}_{1}\right)-\mathrm{z}_{3}^{2}}{\left(\mathrm{z}_{3}-\mathrm{z}_{2}\right)\left(\mathrm{z}_{3}-\mathrm{z}_{1}\right)}
$$

\subsection{Aplicação na vazão regularizada}

A determinação da vazão regularizada do Orós, considerando as duas fontes de informação para a vazão afluente (ANA e COGERH), foi realizada com o objetivo de verificar o impacto que a incerteza investigada pode ocasionar no valor da máxima vazão de retirada. A vazão regularizada é a quantidade de água que pode ser extraída de um reservatório para usos consuntivos com atendimento temporal mínimo de $90 \%$ (HASHIMOTO, STEDINGER e LOUCKS, 1982). A expressão usada para calcular a vazão regularizada é fornecida na Eq. 13.

$$
\mathrm{V}_{\mathrm{t}+1}=\mathrm{V}_{t}+\mathrm{Q}_{\mathrm{a}, \mathrm{t}}-\mathrm{Q}_{\mathrm{r}, \mathrm{t}}-\mathrm{E}_{t} \mathrm{~A}_{t}-\mathrm{S}_{t}
$$

em que $t+1(T)$ e $t(T)$ se referem ao tempo presente e tempo passado, respectivamente, $V\left(L^{3}\right)$ é o volume armazenado, $Q_{r}\left(L^{3} T^{-}\right)$é a vazão regularizada, E (LT') é o vetor da evaporação líquida, A ( $\left.\mathrm{L}^{2}\right)$ é a área da superfície líquida e $S\left(\mathrm{~L}^{3} \mathrm{~T}^{-}\right)$é a vazão vertida. A curva cota-área-volume foi obtida junto no website <http://www.hidro.ce.gov.br/>, o vetor de evaporação líquida do Orós foi adquirido no estudo sobre os reservatórios do semiárido brasileiro realizado pela ANA (ANA, 2017), a vazão proveniente da COGERH foi obtida via solicitação no website <https://www.cearatransparente.ce. gov.br/> e a vazão oriunda da ANA, coletada no website <www.snirh.gov.br/hidroweb/>. As séries históricas da vazão afluente usadas para a COGERH e ANA foram referentes aos períodos 19862016 e 1974-2010, respectivamente.

\section{RESULTADOS E DISCUSSÃO \\ 4.1 Classificação da incerteza}

A incerteza identificada foi classificada como estrutural, segundo a abordagem usada por Gupta e Govindaraju (2019), pois pode ser estabelecida no modelo hidrológico como representativa de toda a sub-bacia. Essa hipótese está presente em muitos estudos que utilizaram a sub-bacia ou o Orós para pesquisas de diversos naturezas, como hidrológica, mudanças climáticas, gestão de recursos hídricos, dentre outros (ARAÚJO et al., 2020; FERNANDES, $R$ et al., 2017; FERNANDES, $W$ et al., 2016; SILVA, SOUZA FILHO e AQUINO, 2017). Talvez a razão dessa simplificação seja a conveniência de considerar o posto de Iguatu como fonte de informação, uma vez que o início das mensurações pelo posto, portanto a construção da série histórica da vazão afluente, foi em 1912, enquanto as do COGERH datam de 1986. Tal hipótese pode fornecer informações hidrológicas que podem afetar a capacidade do sistema hídrico, subestimando ou superestimando o valor da vazão afluente, portanto gerar riscos sobre o sistema (TUNG, 2018). A incerteza global, diferença entre o output do modelo (vazão afluente) e o real valor da variável, assumindo que este é verdadeiro, é intensificada.

Alguns pontos merecem destaque e alerta. Primeiro, a vazão afluente é uma variável hidrológica que, do ponto de vista de construção de sua série histórica, também pode se apresentar como uma incerteza natural e métrica (MCMILLAN, WESTERBERG e KRUEGER, 2018). Campos, Souza Filho e Lima (2014), ao estudarem o risco associado à variabilidade natural da vazão afluente do reservatório Castanhão, localizado na sub-bacia do Médio Jaguaribe, calcularam que a incerteza natural pode aumentar em $25 \%$ o risco de falha na estimativa da vazão regularizada. Por outro lado, Di Baldassarre e Montanari (2009), ao estudarem o Rio Po, Itália, observaram que o erro médio na estimativa da vazão com curva chave pode chegar a $20 \%$. 0 segundo ponto que merece atenção é o procedi- 
mento adotado pela COGERH para a determinação do valor da vazão afluente, balanço hídrico no reservatório, uma vez que possui muitas considerações. As simplificações e hipóteses desse procedimento inserem e propagam várias incertezas até a geração do output final (vazão afluente): incertezas na infiltração, vazão vertida, volume estocado, vazão retirada, percolação através do maciço da barragem (drenagem interna), taxas de evaporação e lâmina de precipitação sobre a superfície do reservatório. Uma inspeção nas duas séries históricas da vazão afluente (1986-2010) revelou uma superioridade da vazão fornecida pela ANA em $22 \%$ das vezes, mesmo com desconsideração de parte da área de drenagem da sub-bacia por essa fonte de dados. Trata-se de um valor relativamente alto, para o qual foram sugeridos alguns motivos: variabilidade espacial da precipitação, forte característica da região semiárida na qual a sub-bacia do Alto Jaguaribe está inserida; erros na medição da vazão pela curva chave, que pode ter superestimado o real valor; e subestimação dos valores pela COGERH em razão das considerações adotadas pelo método do balanço hídrico no reservatório, especialmente quanto às taxas de evaporação e à lâmina de precipitação sobre a superfície do reservatório, uma vez que vazão vertida, volume estocado, vazão retirada são bem controlados (LOUCKS e BEEK, 2005) e as perdas por infiltração e drenagem interna podem ser considerados desprezíveis. Porém, é importante enfatizar que este estudo focou no risco associado à incerteza estrutural, que se estabelece em virtude da razão mencionada anteriormente. Por fim, cabe esclarecer que as investigações realizadas neste trabalho sobre incerteza e risco hidrológico referem-se a hipóteses e simplificações que o modelador poderia assumir sobre a vazão afluente na sub-bacia, em termos de calibração, validação, correção de faIhas da série história e outras aplicações de mode- los hidrológicos, em razão da área desprezada no posto fluviométrico de Iguatu, principal fonte de dados utilizada nos estudos hidrológicos da subbacia do Alto Jaguaribe e do Orós.

\subsection{Capacidades, solicitações e funções- desempenho por meio de NFTs}

A Fig. 4 exibe as representações gráficas da capacidade, solicitação e função-desempenho do sistema estudado, caracterizadas por NFTs.

De maneira geral, a solicitação, a capacidade e a função-desempenho tiveram deslocamentos em torno do eixo das ordenadas de acordo com o mês. Verificou-se que as amplitudes dos elementos característicos dessas funções apresentaram os maiores valores absolutos entre dezembro e maio. Nesses meses, março, maio e dezembro tiveram a função à direita dos pontos de máximo do NFT tocando o eixo das ordenadas. Por outro lado, de junho a novembro as amplitudes dos elementos característicos assumiram os menores valores absolutos. As capacidades dos meses de outubro e novembro exibiram comportamento de fuzzy singleton ${ }^{1}$.

Gupta e Govindaraju (2019) consideram que a caracterização das incertezas estruturais é uma tarefa desafiante. Em bacias semiáridas, a dificuldade é ampliada pela variabilidade espacial e temporal da precipitação (CAMPOS, SOUZA FILHO e LIMA, 2014). A variação do deslocamento da função-desempenho em torno do eixo das ordenadas pode estar associada à sazonalidade da precipitação na sub-bacia, propagada para a vazão afluente. Consistentes com isso, os maiores deslocamentos do ponto de máxima pertinência ocorreram nos meses mais chuvosos do Estado do Ceará. Esses resultados sugerem que a pré-estação, a pós-estação e a estação chu-

\footnotetext{
${ }^{1}$ Conjunto fuzzy formado por um único elemento cujo nivel de pertinência é 1. Nesse caso, pode-se dizer que o conjunto fuzzy é igual ao conjunto crisp. Para mais detalhes, consultar Aliev (2013).
} 
vosa podem ter um impacto significativo na real vazão que chega ao Orós. Observou-se, também, um forte deslocamento para o domínio negativo da função-desempenho nos períodos mais secos, refletindo que nesse período a área desprezada tem um maior impacto sobre a so- licitação do que sobre a capacidade. Verificouse, também, que a função-desempenho fuzzy foi capaz de incorporar e propagar a sazonalidade da vazão afluente, demonstrando ser um método passível de caracterização da incerteza estrutural em bacias semiáridas.
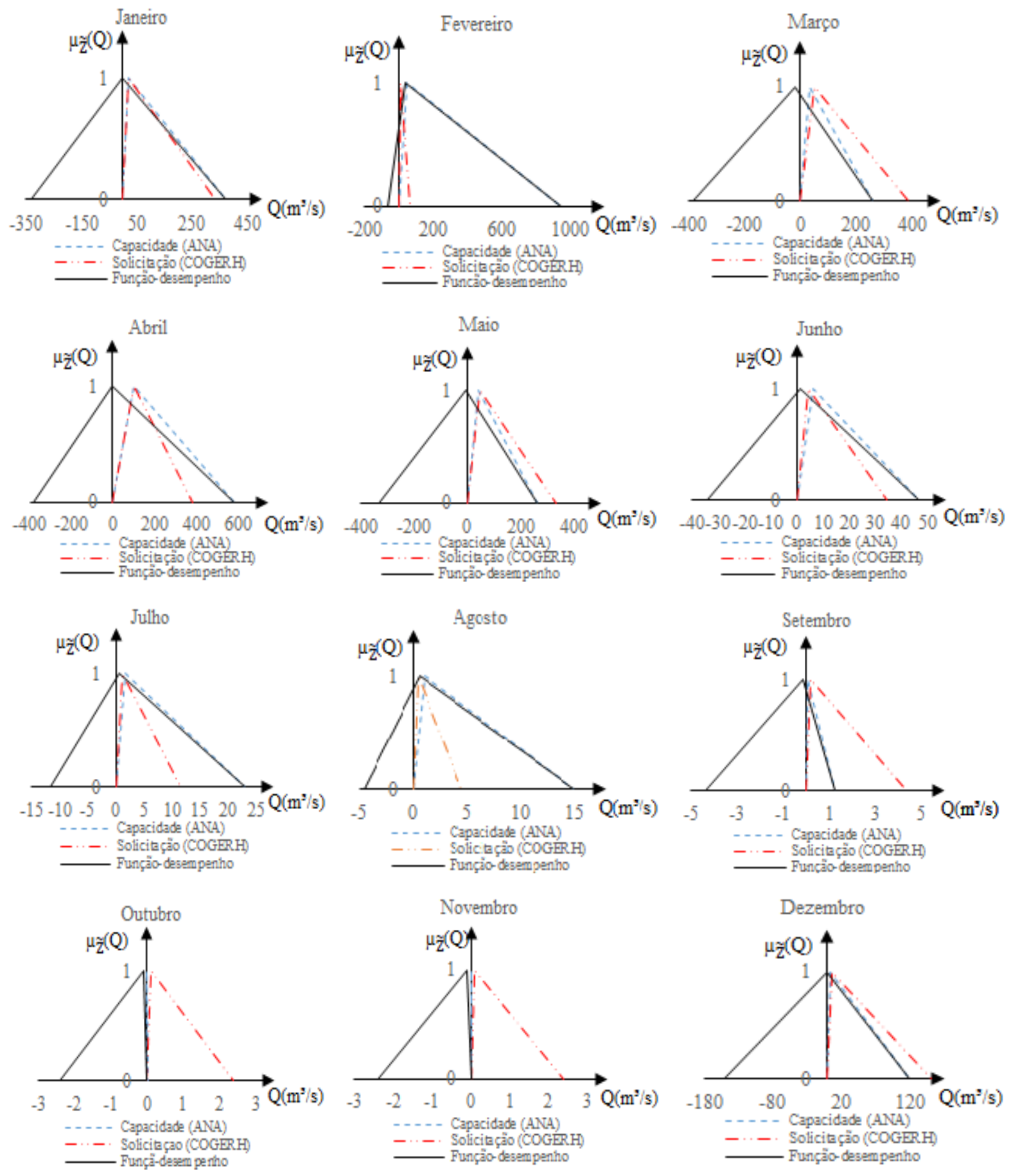

Figura 4 - Definição das capacidades, solicitações e funções-desempenho fuzzy. 


\subsection{Quantificação do risco fuzzy}

A Tabela 1 sintetiza os resultados do cálculo do risco fuzzy mensal da vazão afluente do reserva-

tório Orós. Os riscos mensais variaram ao longo do ano entre $4 \%$ e $100 \%$.

Tabela 1 - Resultados dos riscos fuzzy, arredondados para o valor inteiro mais próximo

\begin{tabular}{|c|c|c|c|c|c|c|c|c|c|c|c|c|}
\hline & \multicolumn{10}{|c|}{ Meses } \\
\hline K(\%) & Jan. & Fev. & Mar. & Abr. & Maio & Jun. & Jul. & Ago. & Set. & Out. & Nov. & Dez. \\
\hline
\end{tabular}

Os riscos fuzzy não nulos em todos os meses constataram que a área desprezada da sub-bacia pelo posto de Iguatu pode contribuir ao longo de todo o ano com produção de água para o Orós. Isso pode ser um problema, se o modelador considerar o período seco do ano não representativo, pois a área desprezada pode ampliar os erros no processo de geração de informações hidrológicas, como no estudo realizado por Araújo et al. (2020). Os valores dos riscos fuzzy permaneceram similares quando realizadas simulações alterando o período de análise, isto é, reduzindo ou aumentado o período usado para o cálculo do risco. 0 risco médio para a pré-estação (novembro e dezembro), pós-estação (junho e julho) e estação chuvosa propriamente dita (fevereiro a maio) foi de $48 \%$, com exceção do mês de fevereiro. A função-desempenho fuzzy foi capaz de identificar essa descontinuidade (Tabela 1), demonstrando sua eficácia para englobar a sazonalidade da vazão afluente. Por outro lado, é preciso ter cautela na interpretação dos altos riscos fuzzy dos meses de setembro, outubro e novembro, especialmente nesses dois últimos. Esses meses estão no período mais seco do ano para a região, de modo que a capacidade (vazão afluente da ANA) se comportou como fuzzy singleton, isto é, como um valor crisp (vazão nula) (Fig. 5). Então, qualquer contribuição da área desprezada pela ANA, mesmo que pequena, ou até a própria vazão nula, matematicamente foi considerada uma faIha total, ou próximo disso. É importante ressaltar que a vazão afluente da COGERH foi tratada como solicitação pelo rigor matemático; no entanto deve também ser interpretado como a capacidade do sistema. Quando as vazões são iguais, especificamente quanto às vazões nulas no período seco, não é uma falha propriamente dita, mas sim uma coincidência entre as capacidades.

Foi observada uma escassez de estudos referentes ao tipo de incerteza identificado neste estudo, o que indica a possibilidade de uma contribuição inédita e de grande importância, especialmente em se tratando de bacias semiáridas, onde a estimativa errônea da disponibilidade hídrica pode afetar a segurança hídrica da região. Outros estudos têm estudado o efeito de outras incertezas epistêmicas sobre os sistemas hídricos, o que pode fornecer a possibilidade de comparações gerais. Este estudo concordou com o de Tung (2018) no sentido de que as incertezas epistêmicas podem impactar no valor da falha do sistema; no entanto, divergiu no sentido do efeito. Nesse mesmo sentido, Qi et al. (2016) identificaram que as incertezas epistêmicas podem influenciar na cheia de projeto de estruturas hidráulica, ou seja, na capacidade desses sistemas hídricos. 0 mesmo foi observado por Moel et al. (2011), ao avaliarem riscos de ondas de cheias em planícies de inundação. De maneira geral, esses estudos concordam que a presença de incertezas epistêmicas, como o caso da incerteza estrutural encontrada neste trabalho, afeta a real capacidade do sistema, impactando a confiabilidade nas informações que auxiliam o gerenciamento de risco dos sistemas hídricos. 
4.4 Verificação dos riscos fuzzy calculados

A Fig. 5 exibe uma comparação entre as vazões afluentes mensais da ANA e COGERH de 2014 a
2019 para corroborar riscos fuzzy mensais calculados para o período 1986-2010. (a)

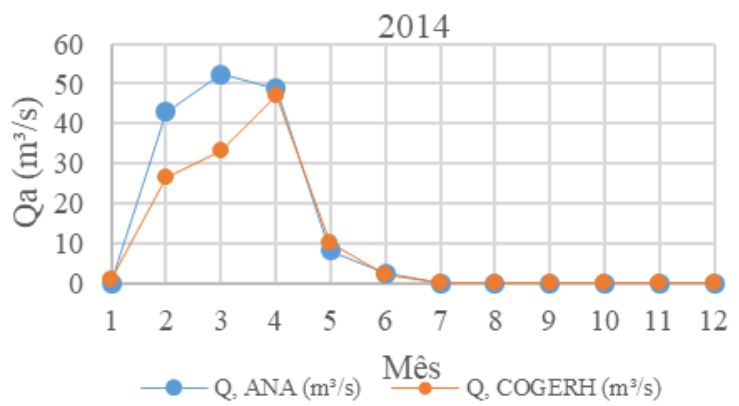

(c)

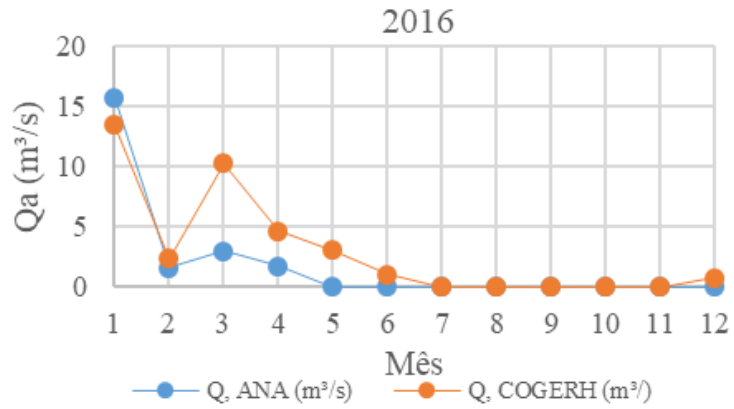

(e)

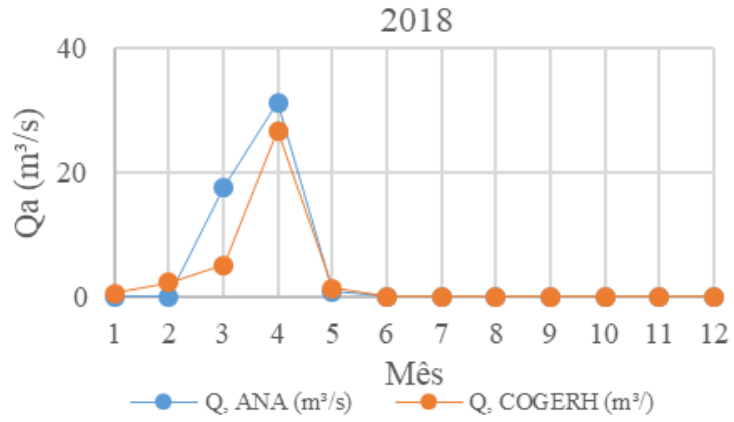

(b)

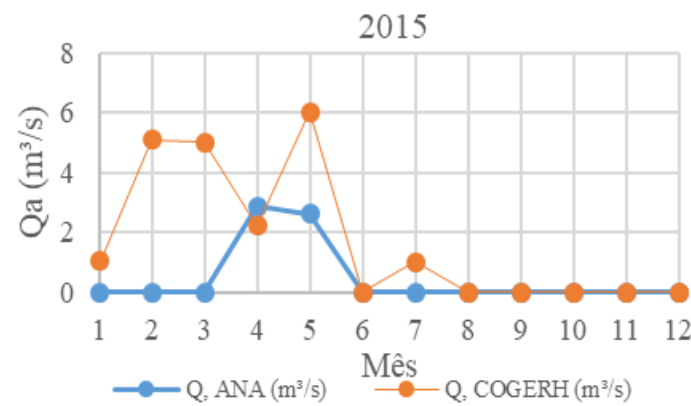

(d)

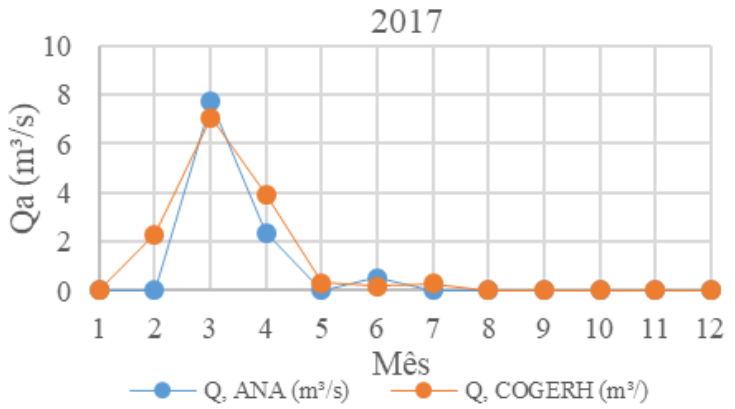

(f)

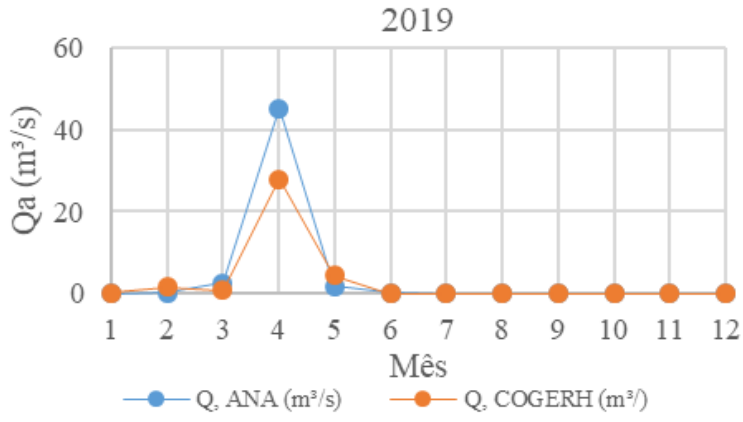

Figura 5 - Comparativo entre as vazões afluentes médias mensais da ANA e COGERH para os anos: (a) 2014, (b) 2015, (c) 2016, (d) 2017, (e) 2018 e (f) 2019.

\subsection{Aplicação na vazão regularizada}

Uma análise realizada sobre a vazão regularizada revelou que o valor gerado a partir dos dados da ANA superestimou a falha em $24 \%$, compa- rada à vazão gerada pela COGERH. Esse resultado indicou que a incerteza estrutural identificada interferiu na real capacidade do sistema hídrico, corroborando com os riscos fuzzy calcu- 
lados (Tabela 1). Marton e Paseka (2017) identificaram também a possibilidade de influência de incertezas na capacidade na operação de reservatórios, ao estudarem incertezas métricas relacionadas a dados operacionais e hidrológicos. Por outro lado, o erro na vazão regularizada em razão da área desprezada pode afetar a geração de informações que auxiliam a gestão de recursos hídricos. $O$ risco aceitável definido no estudo de Silva, Souza Filho e Aquino (2017), para subsidiar a transferência hídrica, pode ter sido superestimado, uma vez que considerou a capacidade do sistema hídrico, Açude Orós, usando os dados oriundos do posto de Iguatu. O impacto também pode ser propagado para as projeções futuras da vazão regularizada em cenários de mudanças climáticas. No entanto, em um estudo realizado por Estácio (2020), avaliaram-se apenas as incertezas relativas aos parâmetros do modelo hidrológico utilizado (Soil Moisture Accounting Procedure - SMAP). Campos, Souza filho e Lima (2014), ao estudarem o Açude Castanhão localizado na sub-bacia Médio Jaguaribe, a montante do Alto Jaguaribe, observaram que a variabilidade natural das vazões do Rio Jaguaribe aumentou em $25 \%$ o risco de falha da vazão regularizada de $25 \%$. Esse resultado sugere que a incerteza estrutural identificada no presente trabalho pode ser tão importante quanto a incerteza aleatória, o que merece ser investigado em estudos futuros.

\section{CONCLUSÕES}

Este estudo investigou a aplicabilidade dos NFTs para caracterizar incertezas e quantificar riscos hidrológicos. O estudo de caso utilizado foi o Açude Orós, no Estado do Ceará, que, em razão de duas fontes distintas de informação da vazão afluente, uma que considera toda a área de drenagem da sub-bacia e outra que despreza uma parcela (20\%), gerou uma incerteza classificada como estrutural. Como essa incerteza poderia ser relevante na ge- ração de informação hidrológica e capaz de afetar a real capacidade do sistema hídrico, foi necessário avaliar o risco associado. A capacidade e a solicitação do sistema foram definidas por meio de NFTs, usando a série histórica do período 1986-2010 e as características da região no tocante à sazonalidade da vazão, em virtude dos períodos chuvoso e seco do Estado do Ceará. As funções-desempenho mensais apresentaram diferentes comportamentos de acordo com o mês, acompanhando o padrão da vazão afluente da região. A metodologia com base nos NFTs proposta foi capaz de incorporar e propagar a sazonalidade da vazão afluente na região estudada, demonstrando ser um método passível de caracterização da incerteza estrutural em bacias semiáridas. Os resultados exibiram riscos não nulos em todos os meses do ano, demonstrando que a área desprezada pode contribuir com a produção de água para o Orós durante todo o ano. Os riscos fuzzy foram maiores no período seco, com exceção de agosto, enquanto na pré-estação, pósestação e estação chuvosa teve um risco médio de $48 \%$. No exemplo numérico para ilustrar o impacto da incerteza estrutural, vazão regularizada, observou-se um impacto significativo sobre a real capacidade do sistema hídrico. O risco de falha da vazão regularizada, determinada a partir dos dados da ANA, foi $24 \%$ maior dos que os $10 \%$ usados para estimar a vazão regularizada, quando comparado com a vazão regularizada gerada a partir dos dados da COGERH. Esse resultado indicou que a incerteza estrutural identificada interferiu na real capacidade do sistema hídrico, corroborando os riscos fuzzy calculados. De modo geral, demonstrou-se a aplicabilidade da metodologia com base nos números fuzzy para caracterizar a incerteza estrutural do sistema avaliado e quantificar o risco associado.

\section{AGRADECIMENTOS}

À Fundação Cearense de Apoio ao Desenvolvimento Científico e Tecnológico - FUNCAP (Projeto PRONEM/FUNCAP/CNPq/PNE0112-00042.01.00/16). 


\section{CONTRIBUIÇÃO DOS AUTORES}

\section{Contextualização: Raulino JBS; Metodologia:} Raulino JBS; Análise formal: Raulino JBS; Escrita: Raulino JBS, Malveira VTC, Lima Neto IR; Revisão \& Edição: Raulino JBS, Malveira VTC, Lima Neto IR; Desenvolvimento do estudo de caso: Malveira VTC; Supervisão: Malveira VTC, Lima Neto IR; Conselhos: Malveira VTC, Lima Neto IR; Problemática: Lima Neto IR.

\section{REFERÊNCIAS}

AHMADI, A.; NASSERI, M.; SOLOMATINE, D. P. Parametric uncertainty assessment of hydrological models: coupling UNEEC-P and a fuzzy general regression neural network. Hydrological Sciences Journal. v. 64, n. 9, p.1080-1094, 2019. https://doi.org/10.1080/ 02626667.2019 .1610565

ALIEV, R. A. Fundamentals of the Fuzzy Logic-Based Generalized Theory of Decisions. Berlin: Springer, 2013.

ANA. Ministério do Desenvolvimento Regional. Agência Nacional de Águas e Saneamento básico. Reservatórios do semiárido brasileiro: hidrologia, balanço hídrico e operação. Brasília, DF, 1ed, 2017.

ARAÚJO, C. B. C.; SOUZA FILHO, F. A.; ARAÚJO JÚNIOR, L. M.; SILVEIRA, C. S. Previsão sazonal de vazões para o Bacia do Orós/CE (Ceará, Brasil) utilizando redes neurais e a técnica de reamostragem dos K-vizinhos. Revista Brasileira de Meteorologia. v. 34, n. 2, p.197-207, 2020. https://doi.org/10.1590/0102-7786351015

BOUDA, M.; ROUSSEAU, A. N.; KONAN, B., GAGNON, P.; GUMIERE, S. J. Bayesian uncertainty analysis of the distributed hydrological model HYDROTEL. Journal of Hydrologic Engineering. v. 17, $\mathrm{n}$. 9, p.1021-1032, 2012. https://doi.org/10.1061/(ASCE)HE.19435584.0000550

CAMPOS, J. N. B.; SOUZA FILHO, F. A.; LIMA, H. V. C. Risks and uncertainties in reservoir yield in highly variable intermittent rivers: case of the Castanhão Reservoir in semi-arid Brazil. Hydrological Sciences Journal. v. 54, n. 6, p.1184-1195, 2014. https://doi.org/ $10.1080 / 02626667.2013 .836277$

CHACHI, J.; TAHERI, S. M.; ARGHAMI, N. R. A hydrid fuzzy regression model and its application in hydrology engineering. Applied Soft Computing. v. 25, n. 1, p.149-158, 2014. https://doi.or$\mathrm{g} / 10.1016 /$ j.asoc.2014.09.010

COGERH. Secretaria dos Recursos Hídricos. Companhia dos Recursos Hídricos do Estado do Ceará. Inventário Ambiental do Açude Orós. Fortaleza, Ceará, 2011. Disponível em: https://portal. cogerh.com.br/wpcontent/uploads/pdf/inventarios/2011/Inven-
tario\%20Ambiental\%20do\%20Acude\%200ros\%202011.pdfao. Acesso em: 22 de julho de 2020.

Di BALDASSARRE, G.; MONTANARI, A. Uncertainty in river discharge observations: a quantitative analysis. Hydrology and Earth System Sciences. v. 6, n. 1, p. 913-921, 2009. https://doi. org/10.5194/hess-13-913-2009

ENGELAND, K.; STEINSLAND, I.; JOHANSEN, S. S.; PETERSEN-OVERLEIR, A.; KOLBERG, S. Effects of uncertainties in hydrological modelling. A case study of a mountainous catchment in Southern Norway. Journal of Hydrology. v. 536, n. 1, p.147,160, 2016. https://doi.org/10.1016/j.jhydrol.2016.02.036

ESTÁCIO, A. B. S. Climate change and model parameter uncertainties propagated to ungauged reservoir catchments in Ceará: a study for water availability assessment. Dissertação (Mestrado) - Programa de Pós-graduação em Engenharia Civil (Recursos Hídricos), Departamento de Engenharia Hidráulica e Ambiental, UFC. Fortaleza, Ceará, 2020.

FERNANDES, R. O.; SILVEIRA, C. S.; STUDART, T. C.; SOUZA FILHHO, F. A. Reservoir yield intercomparison of large dams in Jaguaribe Basin-CE in climate change scenarios. Revista Brasileira de Recursos Hídricos. v. 22, n. 11, 2017. https://doi.org/10.1590/2318-0331.011716033. Disponível em: https://www.scielo.br/scielo.php?script=sci_arttext\&pid=S2318-03312017000100225\&lng=en\&tlng=en. Acesso em 22 de julho de 2020.

FERNANDES, W. S.; SOUZA FILHO, F. A.; STUDART, T. M.; SILVEIRA, C. S. Avaliação do impacto das mudanças climáticas no balanço hídrico do Orós usando os modelos de mudanças climáticas do IPCC-AR4 para o cenário 1B. Revista AIDIS de Ingeniería y Ciencias Ambientales. v. 9, n. 1, p.28-48, 2016. http://dx.doi. org/10.22201/iingen.0718378xe.2016.9.1.49791

GAN, Y.; LIANG, X. Z.; DUAN, Q.; YE, A.; DI, Z.; HONG, Y.; LI, J. A systematic assessment and reduction of parametric uncertainties for a distributed hydrological model. Journal of Hydrology. v. 564, n. 1, p. 697-711, 2018. https://doi.org/10.1016/j.jhydrol.2018.07.055

GANOULIS, J. G. Risk analysis of water pollution. 2. ed. New York: WILEY VCH, 2009.

GUPTA, A.; GOVINDARAJU, R. S. Propagation of structural uncertainty in watershed hydrologic models. Journal of Hydrology, v. 575, n. 1, p.66-81, 2019. https://doi.org/10.1016/j.jhydrol.2019.05.026

HASHIMOTO, T.; STEDINGER, J. R.; LOUCKS, D. P. Reliability, resiliency, and vulnerability criteria for water resource system performance evaluation. Water Resources Research. v. 18, n. 1, p.1420, 1982. https://doi.org/10.1029/WR018i001p00014

HUANG, Y.; CHEN, X.; LI, Y. P.; HUANG, G. H.; LIU, T. A fuzzy-based simulation method for modelling hydrological processes under uncertainty. Hydrological Processes. v. 24, n. 25, p.3718-3732, 2010. https://doi.org/10.1002/hyp.7790 
JACQUIN, A. P.; SHAMSELDIN, A. Development of a possibilistic method for the evaluation of predictive uncertainty in rainfall-runoff modeling. Water Resources Research. v. 43, n. 4, W04425, 2007. https://doi.org/10.1029/2006WR005072

KAVACAN, E.; KHANESAR, M. A. Fuzzy neural networks for real time control applications: concepts, modeling and algorithms for fast learning. UK: Elsevier, 2016.

KESKIN, M. E.; TAYLAN, D.; TERZI, O. Adaptive neural-based fuzzy inference system (ANFIS) approach for modelling hydrological time series. Hydrological Sciences Journal. v. 51, n. 4, p.588-598, 2013. https://doi.org/10.1623/hysj.51.4.588

KHAZAEI, B.; HOSSEINI, S. M. Improving the performance of water balance equation using fuzzy logic approach. Journal of Hydrology. v. 524, n. 1, p.538-548, 2015. https://doi.org/10.1016/j.jhydrol.2015.02.047

LIMA NETO, I. E.; WIEGAND, M. C.; ARAÚJO, J. C. Sediment redistribution due to a dense reservoir network in a large semi-arid Brazilian basin. Hydrological Sciences Journal. v. 56, n. 2, p.319-333, 2011. https://doi.org/10.1080/02626667.2011.553616

LIU, Y.; GUPTA, H. Uncertainty in hydrologic modeling: toward an integrated data assimilation framework. Water Resources Research. v. 43, n. 7, p.1-18, 2007. https://doi.org/10.1029/2006WR005756

LIU, Y. R.; LI, Y. P.; HUANG, G. H.; ZHANG, J. L.; FAN, Y. R. A Bayesian-based multilevel factorial analysis method for analyzing parameter uncertainty of hydrological model. Journal of Hydrology. v. 553, n. 1, p.750-762, 2017. https://doi.org/10.1016/j.jhydrol.2017.08.048

LOUCKS, D. P.; BEEK, E. V. Water resources systems planning and management: an introduction to methods, models and applications. Paris: UNESCO, 2005.

MAMDANI, E. H. Application of fuzzy algorithms for control simple dynamic plant. PROCEEDINGS IEE. v. 121, n. 12, p.1585-1588, 1974. http://dx.doi.org/10.1049/piee.1974.0328

MARTON, D.; PASEKA, S. Uncertainty impact on water management analysis of open water reservoir. Environments. v. 4, n. 1, p.1-14, 2017. https://doi.org/10.3390/environments 4010010

MCMILLAN, H. K.; JACKSON, B.; CLARK, M.; KAVETSKI, D.; WOODS, R. Rainfall uncertainty in hydrological modelling: an evaluation of multiplicative error models. Journal of Hydrology, v. 400, n. 1-2, p. 83-94, 2011. https://doi.org/10.1016/j.jhydrol.2011.01.026

McMILLAN, H. K.; WESTERBERG, I. K.; KRUEGER, T. Hydrological data uncertainty and its implications. WIREs WATER. v. 5, n. 6 , p.1-14, 2018. https://doi.org/10.1002/wat2.1319

MOEL, H.; ASSELMAN, N. E. M.; AERTS, J. C. J. H. Uncertainty and sensitivity analysis of coastal flood damage estimates in the west of the Netherlands. Natural Hazards and Earth System
Sciences. v. 12, n. 4, 1045-1058, 2012. https://doi.org/10.5194/ nhess-12-1045-2012

MONTANARI, A. Uncertainty of hydrological predictions. In: WILDERER, P., organizador. Treatise on Water Science, vol. 2. Oxford: Academic Press; 2011. p.459-478.

NASSERI, M.; ANSARI, A.; ZAHRAIE, B. Uncertainty assessment of hydrological models with fuzzy extension principle: evaluation of a new arithmetric operator. Water Resources Research. v. 50, n. 2, p.1095-1111, 2014. https://doi.org/10.1002/2012WR013382

QI, W.; ZHANG, C.; FU, G.; ZHOU, H. Imprecise probabilistic estimation of design floods with epistemic uncertainties. Water Resources Research. v. 52, n. 6, p.4823-4844, 2016. https://doi. org/10.1002/2015WR017663

SILVA, S. M. O.; SOUZA FILHO, F. A.; AQUINO, S. H. S. Avaliação do risco da alocação de água em período de escassez hídrica: o caso do sistema Jaguaribe-Metripolitano. Revista Brasileira de Engenharia Ambiental e Sanitária. v. 22, n. 4, p.749-760, 2017. http:// dx.doi.org/10.1590/s1413-41522017161303

SIMÕES, M. G.; SHAW, I. S. Controle e modelagem fuzzy, 2. ed. São Paulo: Blucher, 2007

SORDO-WARD, A.; GRANADOS, I.; MATIN-CARRASCO, F.; GARROTE, L. Impact of hydrological uncertainty on water management decisions. Water Resources Management. 30, p. 5535-5551, 2016. https://doi.org/10.1007/s11269-016-1505-5

RENARD, B.; KAVETSKI, D.; KUCZERA, G.; THYER, M.; FRANKS, S. W. Understanding predictive uncertainty in hydrologic modeling: the challenge of identifying input and structural errors. Water Resources Research. v. 46, n. 5, 1-22, 2010. https://doi. org/10.1029/2009WR008328

TAGEGNE, G.; KIM, Y. O.; SEO, S. B.; KIM, Y. Hydrological modelling uncertainty analysis for different flow quantiles: a case study in two hydro-geographically different watersheds. Hydrological Sciences Journal. v. 64, n. 4, p. 473-489, 2019. https://doi.org/10 $.1080 / 02626667.2019 .1587562$

TUNG, Y. K. Effect of uncertainties on probabilistic-based design capacity of hydrosystems. Journal of Hydrology. v. 557, n. 66, p.851-867, 2018. https://doi.org/10.1016/j.jhydrol.2017.12.059

TUNG, Y, K.; YEN, B. C.; MELCHING, C. Hydrosystems engineering reliability assessment and risk analysis. New York: McGraw-Hill, 2005.

VALLAM, P.; QIN, X. S.; YU, J. J. Uncertainty quantification of hydrologic model. APCBEE Procedia. v. 10, n. 1, p.219-223, 2014. https://doi.org/10.1016/j.apcbee.2014.10.042

VIEIRA, V. P. P. B. Análise de risco em recursos hídricos: fundamentos e aplicações. Porto Alegre: ABRH, 2005.

WAGENA, M.; BHATT, G.; BUELL, E.; SOMMERLOT, A. R.; FUKA, D. R.; EASTON, Z. M. Quantifying model uncertainty using Bayesian multi-model ensemnles. Environmental Modelling \& Sof- 
tware. v. 117, p.89-99, 2019. https://doi.org/10.1016/j.envsoft.2019.03.013

WANG, C. X.; LI, Y. P.; ZHANG, J. L.; HUANG, G. H. Assessment parameter uncertainty in semi-distributed hydrological model based on type-2 fuzzy analysis: a case study of Kaidu River Basin. Hydrology Research. v. 46, n. 6, p.969-983, 2015. https://doi. org/10.2166/nh.2015.226

YEVJEVICH, V. 1983. Risk and uncertainty in water resources planning and operation. In: Scientific Procedures Applied to the Planning, Design and Management of Water Resources Systems (Proceedings of the Hamburg Symposium, August 1983). Hamburgo, Alemanha, 1983.
ZADEH, L. A. Fuzzy sets. Information and Control.v. 8, n. 3, p.338353, 1965. https://doi.org/10.1016/S0019-9958(65)90241-X

ZAKHROUF, M.; BOUCHELKIA, H.; STAMBOUL, M. Neuro-Wavelet (WNN) and Neuro-Fuzzy (ANFIS) systems for modeling hydrological time series in arid areas. A case study: the catchment of Ain Hadjadj (Algeria). Desalination and Water Treatment. v. 57, n. 37, p.1-13, 2015. https://doi.org/10.1080/19443994.2015.1085908

ZIMMERMANN, H. J. Fuzzy set theory and its applications. New York: Spriner, 2001.

ZHU, Y. Y.; ZHOU, H. C. Rought fuzzy inference model and its application in multi-factor medium and long-term hydrological forecast. Water Resources Management. v. 23, p.493-507, 2009. https://doi.org/10.1007/s11269-008-9285-1 